Pacific Journal of Mathematics

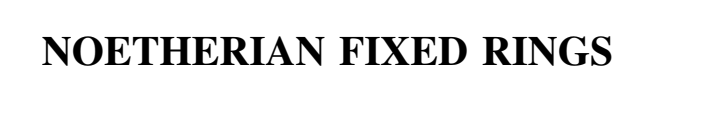




\section{NOETHERIAN FIXED RINGS}

\section{DANIEL R. FARKaS AND Robert L. SNIDER}

One of the basic questions of noncommutative Galois theory is the relation between a ring $R$ and the ring $S$ fixed by a group of automorphisms of $R$. This paper explores what happens when the group is finite and the fixed ring $S$ is assumed to be Noetherian. Easy examples show that $R$ may not be Noetherian; however, in this paper it is shown that $R$ is Noetherian with some rather natural assuptions. More precisely we prove the Theorem 2: Let $S$ be a semiprime ring. Assume that $G$ is a finite group of automorphisms of $S$ and that $S$ has no $|G|$-torsion. If $S^{\theta}$ is left noetherian then $S$ is left noetherian.

Theorem 2 answers a question raised by Fisher and Osterburg [4]. This result rests on calculations which can best be described as belonging to noncommutative Galois theory. The basic theorem here may be of independent interest.

THEOREM 1. Let $R$ be a semisimple artinian ring. If $G$ is a finite group of automorphisms of $R$ and $|G|$ is invertible in $R$ then $R$ is a finitely generated ring $R^{G}$-module.

The proof of Theorem 1 follows the spirit of Karchenko's work on polynomial identity rings ([6]).

1. A proof of Theorem 1. We will repeatedly need Levitzki's fixed ring theorem ([8]): Suppose $R$ is a semisimple artinian ring. If $G$ is a finite group of automorphisms of $R$ with $|G|$ invertible in $R$ then $R^{G}$ is semisimple artinian.

LEMma 1. If Theorem 1 is true when $G$ is a simple group then it is true for an arbitrary finite $G$.

Proof. By induction on the length of a composition series for $G$.

If $G$ is not already simple choose $H \Delta G$ with $1 \neq H \neq G$. By Levitzki's theorem $R^{H}$ is semisimple artinian. $G / H$ acts on $R^{H}$ and $R^{H}$ has no $|G / H|$-torsion; by induction $R^{H}$ is a finitely generated right $R^{G}$-module. Again, induction shows that $R$ is a finitely generated right $R^{H}$-module. The lemma follows.

We eventually assume that $G$ is simple. In that case either $G$ consists entirely of outer automorphisms or entirely of inner automorphisms. 
Lemma 2. Let $B$ be a simple artinian ring and let $G$ be a finite group of outer automorphisms of $B$. Then $B$ is a finitely generated right $B^{G}$-module.

Proof. By [1], $B^{G}$ is a simple ring and $B$ is a free module over $B^{G}$ of rank $|G|$. (Cf. [5] for the case of a division ring.)

LeMma 3. Let $B$ bé a simple artinian ring and let $G$ be a finite group of inner automorphisms of $B$. Assume $|G|$ is invertible in $B$. Then $B$ is a finitely generated right $B^{G}$-module.

Proof. Let $F$ be the center of $B$.

For each $g \in G$ pick one $x \in B$ such that ${ }^{g} b=x b x^{-1}$ for all $b \in B$. Call the finite set so chosen, $\bar{G}$. Then collection of sums, $F \bar{G}$, is a finite dimensional algebra over $F$. Since $1 /|G| \in F$, Maschke's theorem for twisted group algebras ([9]) states that $F \bar{G}$ is a separable algebra. Thus there is a finite extension field $K$ of $F$ such that $K$ is a splitting field for each simple constituent of $F \bar{G}$.

$K \otimes_{F} B$ is a simple artinian ring with center $K . G$ acts on $K \otimes_{F} B$ by

$$
{ }^{g}(k \otimes b)=k \otimes{ }^{g} b
$$

Obviously this action, too, is induced by inner automorphisms. A straight-forward calculation shows that $(K \otimes B)^{G}=K \otimes B^{G}$. Similarly, if $K \otimes B$ is a finitely generated right $(K \otimes B)^{\sigma}$-module then $B$ is a finitely generated $B^{G}$-module.

Thus we replace $B$ with $K \otimes_{F} B$ and assume each simple constituent of $F \bar{G}$ is a total matrix ring with entires in $F$. Let $\mathscr{E}$ be the set of centrally primitive idempotents in $F \bar{G}$.

The crux of this lemma is to show that if $e \in \mathscr{E}$ then $e B e$ is a finitely generated right $B^{G}$-module. An element of $B^{G}$ commutes with elements of $F \bar{G}$ so it certainly commutes with $e$; hence $e B e$ is a right $B^{G}$-module. Let $\varepsilon_{i j}$ be a set of matrix units for $e F \bar{G}$. If $x$ is in $e B e$, set

$$
\pi_{i j}(x)=\sum_{k} \varepsilon_{k i} x \varepsilon_{j k}
$$

$\pi_{i j}(x)$ commutes with each of the matrix units. Since $F$ is the center of $B$, it commutes with $e F \bar{G}$. Thus it commutes with $F \bar{G}$. In other words, $\pi_{i j}(x)$ is in $B^{G}$. The map $\pi_{i j}: e B e \rightarrow B^{G}$ is a right $B^{G}$-module map by the argument at the beginning of this paragraph. We claim that the map

$$
\sum_{i, j} \pi_{i j}: e B e \longrightarrow \bigoplus_{i, j} \sum B^{G}
$$


is injective. For if $\sum_{k} \varepsilon_{k i} x \varepsilon_{j k}=0$ for all $i$ and $j$, multiple on the right by $\varepsilon_{i j}$ :

$$
\varepsilon_{i i} x \varepsilon_{j j}=0 \text { for all } i \text { and } j .
$$

Hence $e x e=0$. But $x \in e B e$ implies $e x e=x$. We finish this paragraph by noticing that Levitzki's theorem says that $B^{G}$ is right noetherian. Since $e B e$ is isomorphic to a submodule of a finitely generated $B^{G_{-}}$ module, $e B e$ is finitely generated.

Next we show that if $e$ and $f$ are different elements of $\mathscr{E}$ then $f B e$ is a finitely generated right $B^{G}$-module. (Of course it is a $B^{G}$ module as above.) Since $B$ is simple, $B e B=B$. Thus we can choose $v_{i} \in f B e$ and $u_{i} \in e B f$ so that

$$
f=\sum_{i} v_{i} u_{i}
$$

Define $\varphi: f B e \rightarrow \oplus \sum_{i} e B e$ by $\varphi(y)=\left(u_{i} y\right)$, a right $B^{G}$-module map. $\varphi(y)=0 \Rightarrow u_{i} y=0$ for each $i \Rightarrow\left(\sum v_{i} u_{i}\right) y=0 \Rightarrow f y=0$. But $f y=y$. Hence $\phi$ is injective. Finish the argument as before.

Because $B=\sum_{e, f \in \varepsilon} f B e, B$ is a finitely generated right $B^{G}$-module.

Proof of Theorem 1. Induct on the order of $G$. Assume $G$ is simple.

Let $e$ be a centrally primitive idempotent in $R . \quad e R$ is a simple artinian ring. Moreover the stabilizer $H=\operatorname{Stab}_{G}(e)$ acts on $e R$ and $1 /|H| e \in e R$. By Lemmas 2 and $3, e R$ is a finitely generated right $(e R)^{H}$-module.

Claim. $(e R)^{H}=e\left(R^{G}\right)$.

Certainly $e\left(R^{G}\right) \leqq(e R)^{H}$. Let $G=\bigcup_{r \in \Gamma} \gamma H$ be a coset decomposition of $G$ with $1 \in \Gamma . G$ permutes the centrally primitive idempotents of $R$ and for $\alpha \neq \beta$ in $\Gamma,{ }^{\alpha} e \neq{ }^{\beta} e$. Equivalently, if $\gamma \neq 1$ is in $\Gamma$, $e\left({ }^{r} e\right)=0$. If $x \in(e R)^{H}$ define $t_{\Gamma}(x)=\sum_{\gamma \in \Gamma}\left({ }^{r} x\right)$. If $g \in G,\{g \gamma \mid \gamma \in \Gamma\}$ are also coset representatives for $H$. Thus ${ }^{g} t_{\Gamma}(x)=t_{\Gamma}(x)$. That is, $t_{T}(x) \in R^{G}$. But $e t_{T}(x)=x$ by the remarks above about multiplying idempotents. Thus $(e R)^{H} \subseteq\left(e R^{G}\right)$.

We now know that $e R$ is a finitely generated right $e\left(R^{G}\right)$-module. That means $e R$ is a finitely generated $R^{G}$-module. Since $R=\sum_{e} e R$, we are done.

\section{Theorem 2 and its relatives.}

Lemma 4. Let $A$ be a semiprime ring. Assume $G$ is a finite group of automorphisms of $A$ and $A$ has no $|G|$-torsion. Then $t_{G}$ does not vanish on any nonzero right ideal of $A$. 


$$
\left(\text { Here } \operatorname{tr}_{G}(\alpha)=\sum_{g \in G}\left({ }^{g} \alpha\right) .\right)
$$

Proof. Suppose $I$ is a right ideal of $A$ with $\operatorname{tr}_{G}(I)=0$. If $J=\sum_{g \in G}{ }^{g} I$ then $J$ is a $G$-invariant right ideal of $A$ with $\operatorname{tr}_{G}(J)=0$. By [2], $J$ is nilpotent. But the only nilpotent right ideal in a semiprime ring is 0 .

Proof of Theorem 2. $S^{G}$ is left Goldie, so according to [6], $S$ is (semiprime) left Goldie. Let $R$ be the left quotient ring for $S$; $R$ is semisimple artinian. By Theorem 1 we can find a finite set of generators $x_{1}, \cdots, x_{n}$ for $R$ as a right $R^{a}$-module. Choose a regular $t$ and $s_{i}$ both in $S$ such that $x_{i}=t^{-1} s_{i}$.

$R=\sum_{i=1}^{n} t^{-1} s_{i} R^{G} \Rightarrow t R=\sum_{i} s_{i} R^{G}$. But $t R=R$ since $t$ is invertible. Thus we assume $x_{i} \in S$.

Define $T: S \rightarrow \bigoplus \sum_{i=1}^{n} S^{G}$ by $T(\alpha)=\left[\operatorname{tr}_{G}\left(a x_{i}\right)\right]_{i=1}^{n}$. $\quad T$ is clearly a left $S^{G}$-module map. We will be done once we prove that $T$ is injective.

$T(a)=0$ implies $t_{G}\left(a x_{i}\right)=0$ for all $i$. But $t r_{G}$ is a right $R^{a_{-}}$ module map. Thus $t r_{G}(a R)=0$. By the previous lemma, $a=0$.

We have actually proved that $S$ is a finitely generated $S^{G}$-module!

One might well ask whether the requirement that $S$ have no $|G|$-torsion can be dropped. Consider the following counterexample. Let $F$ be a field of characteristic $p>2$ and let $\Phi$ be the free group on $x$ and $y$. If $S$ denotes the ring of two-by-two matrices over the group algebra $F[\Phi]$ then $S$ is semiprime but not noetherian. Let $G$ be the multiplicative subgroup of $S$ generated by

$$
\left[\begin{array}{rr}
1 & 0 \\
0 & -1
\end{array}\right],\left[\begin{array}{ll}
1 & 1 \\
0 & 1
\end{array}\right],\left[\begin{array}{ll}
1 & x \\
0 & 1
\end{array}\right] \text {, and }\left[\begin{array}{ll}
1 & y \\
0 & 1
\end{array}\right] \text {. }
$$

$G$ is isomorphic to the semidirect product of $Z / p \oplus Z / p \oplus Z / p$ with $Z / 2$. Since char $F \neq 2, S^{\left[\left[_{0}^{1}-1\right]\right.}$ is the collection of diagonal matrices. The only diagonal matrices fixed by $\left[\begin{array}{ll}1 & 1 \\ 0 & 1\end{array}\right]$ are the scalar matrices. Now a simple calculation shows that $S^{G}$ consists of those scalars in the center of $F[\Phi]$. But it is well known that the center is $F$, a patently noetherian ring.

However, the $|G|$-torsion restriction is not needed when $S$ is (semiprime) commutative or, more generally, when $S$ has no nilpotent elements. There are several difficulties in proving the last statement along the lines of Theorem 2. First, there are division rings on which $\operatorname{tr}_{G}$ vanishes. Even if this objection is met, our induction and restriction techniques all ignore the question of fidelity of action. Reconsider, for instance, Lemma 4. The Bergman-Isaacs theorem states that if $H$ is a group of automorphisms of $J$ and $\operatorname{tr}_{H}(J)=0$ 
then $J=0$. Thus implicit in our argument is the proposition that $\operatorname{tr}_{G}(J)=0 \Rightarrow \operatorname{tr}_{G / K}(J)=0$ where $K$ is the kernel of the action of $G$ on $J$. The implication is true because $J$ has no $|K|$-torsion.

We avoid these complications (and, of course, replace them with other complications) by refining the notion of trace. Let $G$ be a finite group acting on a ring $R$. If $\wedge$ is a subset of $G$ define $t_{\wedge}$ : $R \rightarrow R$ by

$$
t_{\wedge}(r)=\sum_{\lambda \in \wedge}\left({ }^{\lambda} r\right)
$$

$t_{\wedge}$ is an $R^{G}$-bimodule map. Notice that $t r_{G} \equiv t_{G}$.

LEMMA 5. Let $G$ be a finite group acting on the division ring D. Then there is a subset $\wedge \cong G$ such that $t_{\wedge}$ is a mapping from $D$ onto $D^{G}$.

Proof. Suppose we can find $\wedge$ such that $t_{\wedge}$ is a nonzero function from $D$ into $D^{G}$. Say $d \in D$ such that $t_{\wedge}(d)=w \neq 0$. If $x \in D^{G}$, $t_{\wedge}\left(d w^{-1} x\right)=t_{\wedge}(d) w^{-1} x=x$. Thus $t_{\wedge}$ is surjective.

We argue by induction on the length of a composition series for $G$. If $G$ is simple and does not act faithfully then $G$ acts trivially; choose $\wedge=\{1\}$. If $G$ is simple group of automorphisms, a result of Faith ([3]) shows that $t_{G}$ is not identically zero.

When $G$ is not simple choose $H \Delta G$ with $H \neq 1$ and $H \neq G$. By induction there is a subset $A \subseteq H$ such that $t_{A}: D \rightarrow D^{H}$ is surjective. $G / H$ acts on $D^{H}$, so we can find $C \subseteq G / H$ such that $t_{C}: D^{H} \rightarrow D^{G}$ is surjective. If $B$ consists of representatives in $G$ for elements of $C$ then $t_{C}=t_{B}$. Now $t_{B \cdot A}=t_{B} \cdot t_{A}$ is the desired map.

Let $S$ be a ring without nilpotent elements. Suppose $G$ is a finite group of automorphisms of $S$ such that $S^{G}$ is left noetherian. By [7] $S$ is a semiprime left Goldie ring. By the Faith-Utumi theorem the quotient ring, $R$, of $S$ has no nilpotent elements. Let $e$ be a centrally primitive idempotent of $R$.

Lemma 6. $S \cap e R$ is a finitely generated left $S^{G}$-module.

Proof. We first observe that the left quotient ring of $S \cap e R$ in $e R$ is the entire division ring $e R$. Choose $z$ and $s$ in $S$ with $z$ regular such that $e=z^{-1} s$. Then $s=z e \in S \cap e R$. If $x \in e R$ choose $q$ and $w$ in $S$ with $q$ regular such that $q x=w$. Then $(s q) x=s w$. But $s q$ and $s w$ are in $S \cap e R$ with $s q$ regular when considered as an element in $e R$.

$H=\operatorname{Stab}_{G}(e)$ is a group which acts on $S \cap e R$. Pick a transversal, $G=\Gamma \cdot H$. As in Theorem 1 , if $a \in S^{H} \cap e R$ then 


$$
t_{\Gamma}(\alpha) \in S^{G} \text { and } e \cdot t_{\Gamma}(\alpha)=\alpha .
$$

Thus $t_{\Gamma}$ is an injective left $S^{G}$-module map from $S^{H} \cap e R$ into $S^{G}$.

The Galois theory for division rings ([5]) as applied to $e R$ implies that $e R$ is a finite dimensional right $(e R)^{H}$-vector space. As in the proof of Theorem 2 we can choose a basis $x_{1}, \cdots, x_{n}$ in $\mathrm{S} \cap e R$. Use Lemma 5 to find $\wedge \subseteq H$ so that $t_{\wedge}$ is nondegenerate on $e R$. Define $T: \mathrm{S} \cap e R \rightarrow \oplus \sum_{i=1}^{n} S^{G}$ by

$$
T(\alpha)=\left[t_{\Gamma \cdot \wedge}\left(\alpha x_{i}\right)\right]_{i=1}^{n} \text {. }
$$

It is easy to check that $T$ is a well defined left $S^{G}$-module map. The lemma is completed by showing that $T$ is injective. Suppose $a \neq 0$ and $T(a)=0$. Then $t_{\Gamma} \cdot t_{\Lambda}\left(a x_{i}\right)=0$ for each $i$. Since $t_{\Gamma}$ is injective, $t_{\wedge}\left(a x_{i}\right)=0$ for each $i$. That is, $t_{\wedge}(a \cdot e R)=0$. But $e R$ is a division ring: $a \cdot e R=e R$. We have contradicted the nonvanishing of $t_{\wedge}$.

THEOREM 3. Let $S$ be a ring without nilpotent elements. If $G$ is a finite group of automorphisms of $S$ and $S^{G}$ is left noetherian then $S$ is left noetherian (in fact, is finitely generated as an $S^{G_{-}}$ module).

Proof. So far we have proved that $\sum_{e}(S \cap e R)$ is a finitely generated left $S^{G}$-module, where the sum is taken over the centrally primitive idempotents of $R$.

As observed in the first paragraph of Lemma $6, S \cap e R$ contains an element invertible in $e R$. Consequently there is an element $d \in \Sigma(S \cap e R)$ which is invertible in $R$. Define $f: S \rightarrow \Sigma(S \cap e R)$ by $f(s)=s d$. Since $f$ is an injective left $S^{G}$-module map, $S$ is a finitely generated left $S^{\sigma}$-module.

\section{REFERENCES}

1. G. Azumaya and T. Nakayama, On irreducible rings, Ann. of Math., 48 (1947), 949-965.

2. G. M. Bergman and I. M. Isaacs, Rings with fixed-point-free group actions, Proc. London Math. Soc., 27 (1973), 69-87.

3. C. Faith, Galois subrings of Ore domains are Ore domains, BAMS, 78 (1972), 1077-1080.

4. J. W. Fisher and J. Osterburg, Semiprime ideals in rings with finite group actions, to appear.

5. N. Jacobson, Structure of rings, Amer. Math. Soc. Colloq. Publ., 37 (1964).

6. V. K. Kharchenko, Galois extensions and quotient rings, Algebra and Logic (transl.), Nov. 1975, 265-281.

7. V. K. Kharchenko, Generalized identities with automorphisms, Algebra and Logic (transl.), March 1976, 132-148.

8. J. Levitzki, On automorphisms of certain rings, Ann. of Math., 36 (1935), 984-992. 
9. D. S. Passman, Radicals of twisted group rings, Proc. London Math. Soc., (3), 20 (1970), 409-37.

Received October 13, 1976. Farkas was partially supported by NSF grant MCS7606010 and Snider by NSF grant MCS76-05991.

Virginia Polytechnic Institute and State University

BLACKSBURG, VA 24061 



\section{PACIFIC JOURNAL OF MATHEMATICS}

\section{EDITORS}

RICHARD ARENS (Managing Editor)

University of California

Los Angeles, CA 90024

R. A. BEAUMONT

University of Washington

Seattle, WA 98105

C. C. Moore

University of California

Berkeley, CA 94720

\section{J. DugundJI}

Department of Mathematics

University of Southern California

Los Angeles, CA 90007

R. Finn and J. Milgram

Stanford University

Stanford, CA 94305

\section{ASSOCIATE EDITORS}

\section{E. F. BECKENBACH}

B. H. NEUMANN

F. WoLF

K. YOSHIDA

\section{SUPPORTING INSTITUTIONS}

\author{
UNIVERSITY OF BRITISH COLUMBIA \\ CALIFORNIA INSTITUTE OF TECHNOLOGY \\ UNIVERSITY OF CALIFORNIA \\ MONTANA STATE UNIVERSITY \\ UNIVERSITY OF NEVADA \\ NEW MEXICO STATE UNIVERSITY \\ OREGON STATE UNIVERSITY \\ UNIVERSITY OF OREGON \\ OSAKA UNIVERSITY
}

\author{
UNIVERSITY OF SOUTHERN CALIFORNIA \\ STANFORD UNIVERSITY \\ UNIVERSITY OF HAWAII \\ UNIVERSITY OF TOKYO \\ UNIVERSITY OF UTAH \\ WASHINGTON STATE UNIVERSITY \\ UNIVERSITY OF WASHINGTON \\ AMERICAN MATHEMATICAL SOCIETY
}

The Supporting Institutions listed above contribute to the cost of publication of this Journal, but they are not owners or publishers and have no responsibility for its content or policies.

Mathematical papers intended for publication in the Pacific Jaurnal of Mathematics should be in typed form or offset-reproduced, (not dittoed), double spaced with large margins. Please do not use built up fractions in the text of your manuscript. You may however, use them in the displayed equations. Underline Greek letters in red, German in green, and script in blue. The first paragraph or two must be capable of being used separately as a synopsis of the entire paper. Items of the bibliography should not be cited there unless absolutely necessary, in which case they must be identified by author and Journal, rather than by item number. Manuscripts, in triplicate, may be sent to any one of the editors. Please classify according to the scheme of Math. Reviews, Index to Vol. 39. All other communications should be addressed to the managing editor, or Elaine Barth, University of California, Los Angeles, California, 90024.

The Pacific Journal of Mathematics expects the author's institution to pay page charges, and reserves the right to delay publication for nonpayment of charges in case of financial emergency

100 reprints are provided free for each article, only if page charges have been substantially paid. Additional copies may be obtained at cost in multiples of 50 .

The Pacific Journal of Mathematics is issued monthly as of January 1966. Regular subscription rate: $\$ 7200$ a year (6 Vols., 12 issues). Special rate: $\$ 36.00$ a year to individual members of supporting institutions.

Subscriptions, orders for back numbers, and changes of address should be sent to Pacific Journal of Mathematics, 103 Highland Boulevard, Berkeley, California, 94708.

PUBLISHED BY PACIFIC JOURNAL OF MATHEMATICS, A NON-PROFIT CORPORATION

Printed at Kokusai Bunken Insatsusha (International Academic Printing Co., Ltd.). 8-8, 3-chome, Takadanobaba, Shinjuku-ku, Tokyo 160, Japan.

Copyrit (C) 1975 by Pacific Journal of Mathematics Manufactured and first issued in Japan 


\section{Pacific Journal of Mathematics \\ Vol. 69, No. $2 \quad$ June, 1977}

Carol Alf and Thomas Alfonso O'Connor, Unimodality of the Lévy spectral

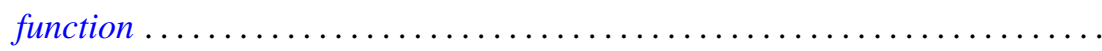

S. J. Bernau and Howard E. Lacey, Bicontractive projections and reordering of

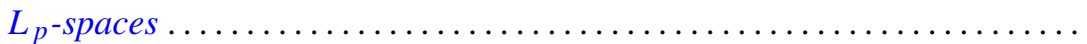

Andrew J. Berner, Products of compact spaces with bi-k and related spaces..... 303

Stephen Richard Bernfeld, The extendability and uniqueness of solutions of ordinary differential equations ...............................

Marilyn Breen, Decompositions for nonclosed planar m-convex sets ..........

Robert F. Brown, Cohomology of homomorphisms of Lie algebras and Lie

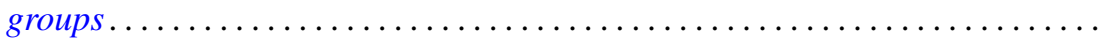

Jack Douglas Bryant and Thomas Francis McCabe, A note on Edelstein's iterative test and spaces of continuous functions ....................

Victor P. Camillo, Modules whose quotients have finite Goldie dimension ....... 333

David Downing and William A. Kirk, A generalization of Caristi's theorem with

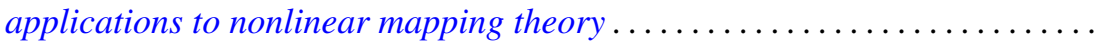

Daniel Reuven Farkas and Robert L. Snider, Noetherian fixed rings ...........

Alessandro Figà-Talamanca, Positive definite functions which vanish at

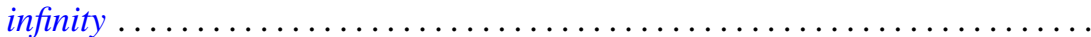

Josip Globevnik, The range of analytic extensions .................. 365

André Goldman, Mesures cylindriques, mesures vectorielles et questions de

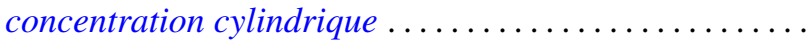

Richard Grassl, Multisectioned partitions of integers..........

Haruo Kitahara and Shinsuke Yorozu, A formula for the normal part of the

Laplace-Beltrami operator on the foliated manifold .... .

Marvin J. Kohn, Summability $R_{r}$ for double series .........

Charles Philip Lanski, Lie ideals and derivations in rings with involution ..

Solomon Leader, A topological characterization of Banach contractions . .

Daniel Francis Xavier O’Reilly, Cobordism classes of fiber bundles . .

James William Pendergrass, The Schur subgroup of the Brauer group . .

Howard Lewis Penn, Inner-outer factorization of functions whose Fourier series

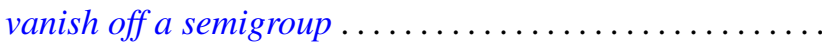

501

William T. Reid, Some results on the Floquet theory for disconjugate definite

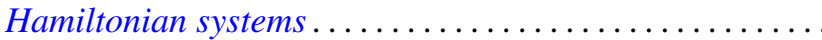

Caroll Vernon Riecke, Complementation in the lattice of convergence

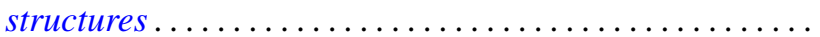

Louis Halle Rowen, Classes of rings torsion-free over their centers ......... 527

Manda Butchi Suryanarayana, A Sobolev space and a Darboux problem ....... 535

Charles Thomas Tucker, II, Riesz homomorphisms and positive linear maps.... 551

William W. Williams, Semigroups with identity on Peano continua ........... 557

Yukinobu Yajima, On spaces which have a closure-preserving cover by finite 